Favaretto, M., Vears, D.F., Borry, P. (2020). On the Epistemic Status of Prenatal Ultrasound: Are Ultrasound Scans Photographic Pictures? JOURNAL OF MEDICINE AND PHILOSOPHY, 45 (2), 231250. doi: $10.1093 / \mathrm{imp} / \mathrm{jhz} 039$

\title{
On the Epistemic Status of Prenatal Ultrasound: are Ultrasound Scans Photographic Pictures?
}

Maddalena Favaretto, Danya F. Vears, Pascal Borry

Centre for Biomedical Ethics and Law, Department of Public Health and Primary Care, Leuven, Belgium.

Corresponding author:

Maddalena Favaretto

Centre for Biomedical Ethics and Law, Department of Public Health and Primary Care Kapucijnenvoer 35

3000 Leuven

Belgium

Phone: +324954327 78 / +393441358536

Mail: favarettomaddalena@gmail.com

\begin{abstract}
Medical imaging is predominantly a visual field. In this context, prenatal ultrasound images assume intense social, ethical, and psychological significance by virtue of the subject they represent: the foetus. This feature, along with the sophistication introduced by threedimensional (3D) ultrasound imaging that allows improved visualization of the foetus, has contributed to the common impression that prenatal ultrasound scans are like photographs of the foetus. In this article we discuss the consistency of such a comparison. First we investigate the epistemic role of both analogic and digital photographic images as visual informationproviding representations holding a high degree of objectivity. Second, we examine the structure and process of production of ultrasound scans, and argue that a comparison between two-dimensional (2D) ultrasound and photography is justified. This is in contrast to 3D ultrasound images that, due to the intensive mathematical processing involved in their production present some structural issues, that obfuscate their ontological and epistemic status.
\end{abstract}


Keywords: photography, medical imaging, three-dimensional ultrasound, epistemic value, ontology.

\section{Introduction}

Ultrasound examination is a widespread procedure in obstetric practice. Its main purpose is to assess the health and growth of the foetus during pregnancy, and to detect markers, which are features of the foetus identified on the ultrasound scan that indicate an increased risk for genetic diseases, or foetal malformations. Aside from its acknowledged diagnostic value, foetal ultrasound is usually highly appreciated by parents who want to visualise their unborn child during pregnancy (Roberts 2012). The distinctive trait of this examination is that it takes place through the production, and subsequent observation, of images of the foetus in the uterus; images in which the observer has the intuitive feeling of actually seeing the child before it is born. Foetal images can have great impact. Firstly, ultrasound images seem to play a prominent role in the development of parenthood and many studies have reported how the visualization of the foetus by the mother through an ultrasound scan can increase the emotional bond between the mother and the child-to-be (Black 1992; Lumley 1990). Secondly, they also play a central role in ethical debates. Visualization of the physicality of the foetus, and observation of its movements, have been used to define the social and individual identity of the foetus (Mills 2011). In the context of the abortion debate, many pro-life associations make extensive use of prenatal ultrasound images with the purpose of criticizing the abortive practice, for it is considered the interruption of a life that we can actually see with our own eyes through an ultrasound scan (Boucher 2004).

Even when produced for purely diagnostic purposes, as images, foetal ultrasound scans are deeply entwined with social, ethical and psychological significance. This is particularly the case in light of the new technologies that are enhancing our ability to see the appearance and behaviours of the foetus inside the womb. Three-dimensional (3D) ultrasound imaging has 
improved significantly over the last years and is increasingly being used in obstetric practice (Sepulveda et al. 2012; Tonni, Castigliego, et al. 2016). Thanks to this imaging technique, we are able to see the foetus in ways that two-dimensional (2D) ultrasound could never accomplish. It allows us to clearly visualize the appearance and behaviours of the foetus while it is still in the womb (Ramón y Cajal and Martinez 2005; Tonni, Grisolia, et al. 2016). There is a widespread tendency both in the literature and by the general public, to refer to ultrasound scans as if they were photographs of the foetus, especially because of the value placed on the subject of the image (Gilbert and Howes-Mischel 2004; Mills 2011; Roberts 2012). However photography and ultrasonography are not the same: they are two very different technologies both in the way they produce their images and also in their purpose. Moreover, a comparison between photography and ultrasound is even more difficult to assess if we consider 3D ultrasonography, a technology that produces very realistic photographic looking images of the foetus. 3D ultrasound scans are produced by means of a highly sophisticated process that diverges from both photography and 2D ultrasound. Therefore, it is necessary to understand the uniqueness and peculiarity of these different technologies to see if any comparison between their produced images is justified. To the best of our knowledge, very few studies have focused on the characterization of ultrasound scans as images. References to this imaging technology have been made when discussing the status of medical images generally (Delehanty 2005; Semczyszyn 2010; Perini 2012b). Nevertheless, this method of analysis seems to fail in properly stressing the differences that occur between ultrasound technology and other medical imaging techniques. As foetal imaging is progressing (Roberts 2012; Edvardsson et al. 2015), a thorough and specific characterization of ultrasound is needed.

Therefore, the goal of this paper is to investigate the epistemic status of ultrasound images in comparison to other images of mechanical production, such as photographs. First, we will analyse the epistemic status of photography, referring to the evolution of digital technologies, 
and considering some of the most renowned theories of photographic epistemology. Secondly, we will explore the status of $2 \mathrm{D}$ ultrasound, and the consistency of the claims that they are photographs (Boucher 2004; Mills 2011; Petchesky 1987). Finally, we address the ontological and epistemic status of 3D ultrasound images. These images are of particular interest because they are produced by means of mathematical processing and represent the foetus as a sophisticated computer animation, which produces some additional epistemic challenges in comparison to $2 \mathrm{D}$ images.

\section{IMAGES, PHOTOGRAPHS AND THEIR EPISTEMIC STATUS}

In our everyday life, vision plays a very important role in our persistent quest for knowledge. Sight is in fact the main sense that we use to investigate, understand and ultimately "know" the world that surrounds us. We are constantly surrounded by images: magazines, newspapers, social media, and websites are overflowing with them. With the aid of photographic technologies we are able to see, extract, and learn information from things that we are not temporally or spatially related to. For this reason, photographs have an epistemic privilege. There is general agreement among authors on the capacity of photographs to deliver objective information about their depicta (Currie 1991; Perini 2012a; Walden 2005; Nanay 2010). But where does this epistemic privilege that is invested in photographs come from? Many authors claim that the value of evidence of photographs arises firstly from the process of production of the image, and the consequent objectivity of the beliefs we adopt from observing such images (Currie 1991; Walden 2005; Walton 1984), and secondly from the type of information about the depicted object that is delivered by the image itself (Meskin and Cohen 2007).

\section{Mechanical objectivity and belief independence}


According to Kendall Walton, photographs are "transparent" as we literally see the object photographed through the photograph itself (Walton 1984). Through his transparency thesis, the plausibility of which has been discussed extensively (Currie 1991; Warburton 1988; Gaut 2008; Martin 1986; Nanay 2010), Walton claims that photographs possess a high degree of realism and objectivity and that this is why when we look at an object in a photograph we feel "close" or in contact with the object depicted. The realism of photographs is primarily due to their process of production, which is automatic, mechanic, and requires very little human intervention. When taking a picture, the photographer triggers a sequence of automated physical and chemical processes that allow light to record an image on photosensitive paper. It could be argued that the photographic process is based on human intervention, in that it is the photographer who chooses what to depict and how to depict it. However, after the photographer's input, the process of production of the image is entirely automated, both in analogic and in digital photography. Moreover, photographs are causally and counterfactually dependent on their subjects in a way that other images, such as paintings and drawings, are not. Had the subject in front of the camera been different in any way (such as shape, position, or visual characteristics) the photograph would have also been different. Additionally, photographs are independent of the beliefs of the photographer about the scene depicted. Once the parameters of the camera are set and the scene is framed, the image will be produced regardless of the photographer's beliefs about the subject. The visual information that is conveyed by a photograph does not come from their mind but from the actual scene. This characteristic is particularly important and it is one of the main reasons why we intuitively believe in the evidential role of photography (Roskies 2007), which has consequences for the beliefs that are generated in the observer when viewing a photograph. As such, the observer will assume that their perceptual beliefs relating to the depicted scene are built on an image that 
is independent of the photographer's beliefs, and that the imagine possesses a high degree of objectivity (Walden 2005).

\section{The Nature of Digital Photography}

In the past fifteen years, digital technologies for image production have been slowly but steadily replacing analogic photography, which introduces new ontological and epistemic challenges in the debate of photographic imagery (Benovsky 2014; Hopkins 2012; Alcaraz 2016). Digital cameras have almost completely replaced traditional film photography. This transition is due to multiple factors, such as the immediacy of the medium (analogic processing takes a long time to develop the image), cost effectiveness, and widespread availability. Currently, almost every electronic device has a built in digital camera, and image production, through the use laptops and printers, makes it much easier to produce the final image on paper.

Despite the fact that this technology has increased our ability to take pictures at any time, it has also introduced some ontological and epistemic challenges to the world of photographic imagery. One of the main issues is the ease with which digital photographs can be manipulated and altered with appropriate software, both in the early stages of image production, before the

image is even created, and after the image is produced, using post-processing software (Benovsky 2014). One could argue that the evolution from traditional to digital photography has compromised the objectivity of photographic images by dramatically diminishing the powerful evidential role of traditional photography to a point where, because we cannot recognise a modified digital photograph from a traditional photograph, we will cease to consider photography as epistemically privileged. Modifications made in digital photography, especially those made in post-production, are in fact difficult to detect, making it problematic to discern between a highly modified image and a traditional unmodified snapshot (Savendoff 1997; Benovsky 2014). While some uncertainty remains in the literature regarding the epistemic and ontological status of digital photography in comparison to traditional photography, it is clear 
that the doubt revolves around the fact that digital photography is potentially, and with minimal effort, incredibly modifiable, allowing for a greater level of human intervention than analogic photography (Alcaraz 2016; Benovsky 2014; Hopkins 2012; Savendoff 1997). Then, it is not the nature or typology of modifications introduced by digital technologies that blurred the epistemic status of digital photography as much as it is the fact that digital imagery allows for a higher and less discernible degree of alteration than analogic photography. At a level of picture taking, digital photography works very similarly to analogic photography: light enters the camera and is recorded on an electronic sensor that grasps the relevant visual information from the scene. The main difference between analogic and digital photography, is that the information is stored and processed in the camera to produce the final image. For this reason it is still consistent to claim that digital photography is a mechanical process that produces images that are causally and counterfactually dependent on the depicted scene. The digital process captures the relevant visual features of the depicted scene in a manner independent of the beliefs of the photographer and is therefore information-providing (Meskin and Cohen 2007).

The issues begin with the digital manipulation of the image. After the picture is taken, the raw data file stored in the camera is digitally processed using sophisticated software that allows for the photographer's intervention, permitting modifications that can 'falsify' the original information captured by the camera (Walden 2005; Benovsky 2014; Hopkins 2012). The question remains, is this really a threat to the epistemic consistency of digital photography? Even in analogic photography there is a level of interference or intentionality that could, intuitively, undermine an image's objectivity, a concept acknowledged by even the authors that primarily account for the mechanical objectivity of photography. Analogic photography is not immediate and there are numerous choices that the photographer can make both before taking the picture (such as the subject, framing, lens, exposure), and also during the development 
process in the dark room (such as what negative to print, what paper to use, how to burn, dodge and crop). All these decisions influence how the final image will look and what it will show.

However there is a consensus in the claim that, despite the fact that analogic photography cannot exist without interference, it still possesses a high degree of objectivity (Walton 1984; Walden 2005; Currie 1991; Meskin and Cohen 2007; Nanay 2010). This claim might sound more compelling if we analyse Scott Walden's account of photographic objectivity. According to Walden's account, there are primary and secondary levels of mental states that are involved in the production of an image. Primary level mental states are those involved in the production of a handmade picture, such that the image is entirely crafted depending on the beliefs and decisions of the designer of the image. This involves the internal processing (i.e. within the mind of the designer) of the input image (i.e. the scene depicted) in order to produce the output image (i.e. the sketch). In contrast, the analogic photographic process only involves mental states at the secondary level of image production, in that although the photographer can alter the output imagine, such as by framing the picture, the production of the image is external to the mind of the photographer thus maintaining causal and counterfactual dependence. This distinction has consequences on the beliefs that the observer will form about the original scene by observing the depiction in the photograph. As Walden states:

[...] a viewer of a typical photograph forms first-order perceptual beliefs about various features of the original scene, and at the same time possesses a second-order belief that those perceptual beliefs were formed on the basis of an image that was formed without primary mental-state involvement. (Walden 2005, 271)

In other words, the observer will know that their perceptual beliefs have a high degree of objectivity because of the mechanical process involved in the image production. The involvement of mental states in a system is not sufficient to undermine its objectivity. Yet with digital photography, the high potential for alterations and interference has dramatically blurred 
the line between primary and secondary mental involvement. One could therefore consider digital photography as a hybrid between analogic photography and hand drawing. At the level of picture-taking, analogic and digital technologies are very similar in that they both only involve mental states at a secondary level. However, in post-processing, digital photography also allows mental involvement at a primary level. The development of the final image is no longer only a matter of filtering, framing or cropping such as in analog photographs. With digital technology, major alterations are possible, such as inserting elements that did not exist in the original scene or changing the shape of the depicted objects to make them aesthetically more pleasing, and these modifications can significantly distort and influence the beliefs of the observer. But how much digital manipulation, retouching and alteration is permissible for a digital photograph to remain an epistemically privileged image? While authors have proposed that there is, in this sense, a limit on the acceptable digital alterations that a photograph can endure whilst maintaining its epistemic value, it is a vague and undetermined one (Benovsky 2014).

Despite these issues, it is consistent to state that even digital photography can be classified as a visual information-providing technology that possesses a high degree of objectivity. Although the image resulting from the digital process has the potential to be dramatically distorted, this does not depend on the technology itself but on the decisions and intentions of the photographer. It is therefore less a matter of how images are produced by digital photographic systems and more a matter of the use that we make of the technology: digital photography can be used like analogic photography (i.e. a technology that produces representations that display reliable 
information about the visual properties of the depicted objects) by limiting the involvement of mental states at a secondary level in the image production ${ }^{1}$.

\section{The peculiarity of photograph's epistemic value}

Cohen and Meskin agree that photographs are a significant source of information. Based on Dretske's account (Dretske 1981), they define information as an "objective, probabilistic, counterfactual-supporting link between two independent events" (Meskin and Cohen 2007, 2). Photographs provide information about the visually detectable properties of the depicted objects because they bear a relation to the object they depict. Photographs include information about shape and size: had those been different then the photographic image would differ. This is true for photographs, regardless of a subject's beliefs or mental state. However, the distinct epistemic value of photographs does not consist solely in their capacity to carry visual information about their depicted subjects; vision through binoculars also carries this information-sharing feature. What makes photography special is the fact that photographic images are spatially agnostic informants: they fail to deliver information about the egocentric location of the objects they depict. For example, in viewing a photograph, the observer will receive information about how the depicted object looks (such as its colour, shape, size etc.) but they will not receive any information about the location of the object with respect to themselves in time or space ${ }^{2}$. Because of this feature, photographic images differ from other sources of visual information, like ordinary vision or observation through visual prosthetics

\footnotetext{
${ }^{1}$ The use that we normally make of photography in our everyday life can be an evidence of this statement. It is true that there is a widespread trend in the use of Photoshop and other post-processing software but everyday camera users tend to apply very few modifications to the final image. The idea of photography as a tool for framing reality remains strong despite this, which is the reason digital photography is most often used as analogic photography.

${ }^{2}$ The reason why photographs fail to deliver egocentric information is because the counterfactuals relevant for this information do not hold: if the spatial relationship between the photograph and the depicted object was to change (e.g. a person that carries a photograph of a loved one in their wallet) the photograph would not consequently change. One might argue that digital technologies integrate information about temporal and physical location of the picture by adding tags. However, this does not count as egocentric information because it would be similar to writing the location and time of the picture on the back of an analogic photograph with a pen.
} 
(e.g. binoculars, telescopes etc.), which carry both visual and egocentric information. This claim might entail that, because of this absence of information, photographs are somewhat an impoverished source of information. However Cohen and Meskin argue that the special epistemic privilege of photographs comes precisely from this absence. In order to deliver visual information, other sources of information (like ordinary vision), also need to deliver egocentric information. The former cannot happen without the latter. The only way in which we could actually have visual information about an object seen through binoculars is to actually locate ourselves spatially with regard to said object. If anything obstructed our view that interrupting the flow of spatial information, then we would cease to receive the visual information. On the other hand, photographs have the capacity to provide visual information without delivering egocentric information. While viewing a photograph, an observer can learn about the visual properties of an object without being spatially related to the object in real time. This makes photographs “[...] epistemically valuable because they constitute a relatively undemanding source of information about the visually accessible properties of objects - one that works even when we lack information about egocentric location" (Cohen and Meskin 2004, 204).

Despite the new epistemic and ontological challenges established by the development of digital photography, it is consistent to claim that photographs are objective representations of the objects they depict: they therefore possess an epistemic privilege in displaying information about the visual properties of the depicted objects. For the sake of simplicity, when talking about photographs we will refer to the analogic use of digital photography (i.e. photographs as causally and counterfactually dependent representation of the object depicted and lacking primary mental state processing).

\section{TWO DIMENSIONAL ULTRASOUND AND PHOTOGRAPHY}


In the literature regarding the social and ethical issues related to obstetric ultrasound, it is not uncommon for authors to address ultrasound scans as photographs (Petchesky 1987; Taylor 2000; Boucher 2004). In doing so, they assume intuitively the similarity between the two typologies of images and treat them consequently in roughly the same way. To give a striking example, in her analysis of the ethical significance of ultrasound images, Catherine Mills identifies the two imaging technologies, ultrasound and photography. Without giving any further structural explanation, she claims that both photography and ultrasound have the tendency to distort reality by framing it in small, defined, and detached pieces (Mills 2011, 108). In this, she quotes Susan Sontag's On Photography by changing the term photography with the term ultrasound: [...] to look at an ultrasound image "is to participate in another person's (or thing's) mortality, vulnerability, mutability" (Mills 2011, 107).

The intention here is not to argue against this long tradition in unifying ultrasound with photography. The analysis made by Mills and other authors is to be integrated into a more ethical and social context that transcends the simple structural characterization of the image. However, even if ultrasound scans can be intuitively considered as photographs of the foetus, they are still medical images produced for diagnostic purposes and manufactured by specific machines that can produce false perceptions of the produced image.

Foetal ultrasound would not be the first time similarities in the appearance of the image have lead to an unsupported identification between photography and medical imagery (Roskies 2007). Images produced in different ways can have very different epistemic values because, as we have seen for photographs, the epistemic status of an image is intrinsically linked to the way the image is produced. It is therefore meaningful, if not essential, to gain a deeper understanding of the comparison between photography and ultrasound in order to see if their epistemic significance is shared and based on common grounds.

\section{Mechanically produced images and mathematically elaborated images}


To address the question about the epistemic value of ultrasound images we might first want to ask a preliminary question: what kind of image is a two-dimensional ultrasound? Laura Perini suggests an interesting distinction in the field of scientific and medical images by introducing the concept of the mechanically produced image (MPI) (Perini 2012b). The essential characteristic of MPIs is that they are the product of automated imaging technologies in which there is a direct interaction between the analysed specimen and the machine. The product of such technology is a visible array (the image), which is displayed, either on a screen or printed on paper. However, not all medical images can be classified as MPIs. Drawings and diagrams fail to meet the criteria of a MPI because they lack that component of automatic production. Yet some incredibly detailed images produced using complex mathematical computations (mathematically elaborated images), such as fMRI (functional Magnetic Resonance Imaging) and PET (Positron Emission Tomography), also do not meet the criteria for MPIs. This is because they are a) images of a process rather than a physical specimen, and therefore fail in delivering the contact between the machine and the specimen, and b) they produce a set of data (not an actual image) that later, after in-depth mathematical processing, are converted into images using arbitrary representational schemes (Perini 2012b). fMRI images for example, are considered to be records of brain activity. However, fMRI machine is not sensitive to brain activity in itself, but it merely records changes in the oxygenation of the blood in the brain that are associated with brain activity (Roskies 2008). In this sense there is no direct contact between brain activity and the fMRI machine as there is instead in imaging technologies such as X-rays or electron micrographs (Perini 2012b).

Ultrasound meets the requirement to be considered an MPI because B-Mode ultrasound uses basic acoustic principles to produce the image. Sound waves (a narrow beam of pulses of very high frequency sound energy from 1 to $10 \mathrm{MHz}$ ) are produced by placing a transducer on the skin of the patient or mother-to-be. These waves travel in straight lines until they hit a medium 
of different density that partially absorbs and partially refracts the waves. In the case of foetal ultrasound, the transducer picks up the echo signal and maps it into luminosity patterns that enable the observer to see a 2D dissected image of the foetus on a monitor. Nowadays, ultrasound scans are stored digitally for further examination. However, the conversion to a different format of image does not change the structural features of the image that remains a visible luminous mapping of the sound waves that hit the transducer. The ultrasound image is thus produced by a fully mechanic and automated process in which there is a direct interaction between the specimen (the foetus), and the machine (the transducer). This mechanical production confers to ultrasound the same additional characteristics that we identified previously with photographs: images from ultrasound are causally and counterfactually dependent on the specimen they depict and they are produced entirely independently of the beliefs of the sonographer (belief independence).

\section{The epistemic value of MPIs}

Having illustrated that ultrasound images bear some structural similarities to photographs, we can see that the epistemic value of ultrasound will not differ significantly from that of photography. As Perini claims, to investigate scientific images (specifically MPIs) is very similar to investigating photos:

\footnotetext{
Marking surfaces with visible light is, after all, only one way of producing images. Contemporary scientists often use technologies that are closely related to photography [...]. Scientists produce images by marking surfaces using electrons, soundwaves, X-rays, and the like. (Perini 2012a, 153-54)
}

As we described, the defining characteristic of MPIs is automatic and mechanical production. This feature is in line with the literature regarding the use of images in science. According to Daston and Galison, mechanical objectivity is the feature that acts as guarantor for the evidential role of images in science (Daston and Galison 2007). Adopting this view, images are created as measurement outcomes in which the specimen naturally (causally and 
counterfactually) inscribes itself on screen or paper. This allows for the epistemic ideal of nonintervention in scientific representation: the more the image is automatically produced the more objective and epistemically relevant it will be.

Each imaging technology produces figures that have a form created by a selective response to a particular feature of the specimen (such as tissue, biological material, bones, blood flow etc.). As a result, MPIs embody "information about the kinds of things the technique is selectively sensitive to" (Perini 2012b, 161) because, through the interaction with the specimen, they produce a two dimensional pattern (the image). In ultrasound imaging, sound waves are selectively sensitive tissue's density and shape. The transducer is exposed to sound waves and designed so that it sends impulses to the monitor to make the image dark in places where there is no detected wave, and bright where the sound waves interact with the specimen's tissue. In this way, the imaging technology produces an image that is determined by the specimen's visual features, such as size and shape, embodying information about it.

Therefore, the epistemic value of ultrasound images (like photographs) come precisely from their capacity to provide objective visual information about the object they represent, in this case the foetus inside the womb. Ultrasound scans also carry the distinctive epistemic characteristic of photography in that they are capable of delivering visual information about the represented foetus in the absence of egocentric information, long after the real time examination the ultrasound is capable of conveying information about the depicted foetus, either through the printed scan or the recording of the ultrasound session (Szabo 2004).

THE STATUS OF THREE DIMENSIONAL SURFACE RENDERED ULTRASOUND SCANS 
3D imaging was introduced into obstetric practice in the early 2000s (Sepulveda et al. 2012). Thanks to its development and sophistication, use of 3D ultrasound technology is quite widespread and is being considered by many practitioners as diagnostic procedure for use in everyday obstetric practice (Tonni et al. 2015).

Although the additional diagnostic value of 3D technology still requires assessment (Hur et al. 2015; Tonni, Grisolia, et al. 2016), the images produced by this technology undoubtedly look astonishingly realistic when compared with 2D ultrasound scans. These images look completely different from regular 2D scans, appearing more like photographs and videos of the foetus inside the womb. This difference is especially striking since the introduction of HDlive imaging software. These images have the capacity to show the surface of the represented foetus, including its limbs and details of its face, and can capture the visual appearances of the subject in a way that resembles an "in the flesh" visual experience (Semczyszyn 2010). Since the novel feature introduced by 3D technology is its extraordinary photographic realism, threedimensional ultrasound scans are particularly appreciated by prospective parents who want the unique experience of "meeting" their baby before it is born. However, due to its higher cost and limited additional diagnostic utility compared with regular 2D sonography, 3D ultrasound is not a widespread procedure in routine antenatal care. As a consequence, to obtain a 3D/4D ultrasound session (i.e. real time 3D ultrasound imaging), parents must turn to the private sector, either by selecting a private gynaecologist and requesting a 3D ultrasound for an additional cost, or by utilising private commercial practices that sell non-diagnostic ultrasound sessions directly to pregnant women. "Keepsake" commercial ultrasound services have become quite common outside of clinical practice in the US, with small offices appearing in malls, airports and train stations. The popularity of these services is spreading and similar services are now emerging all over Europe, including in the UK, the Netherlands, Denmark and Belgium. These 
non-diagnostic ultrasound images and recorded 3D real time sessions are cherished by parents and are often considered the "baby's first photographs”. (Lubell 2004; Roberts 2012).

Although these 3D images give the impression of photographs, in reality, they greatly differ from both photographs and 2D ultrasound. This is because they are produced by very different techniques. $3 \mathrm{D}$ ultrasound works very similarly to $2 \mathrm{D}$ at the level of data acquisition where multiple bi-dimensional parallel sections of the foetus are obtained by means of a specially designed volumetric transducer that uses the same sound waves involved in 2D ultrasound. However, this is where the similarity between 2D and 3D ends. Once obtained, the data is stored and processed using sophisticated volume rendering software to produce the 3D image (Semczyszyn 2010; Sepulveda et al. 2012). As described previously, in 2D ultrasound, translation of the signal into an image is not particularly problematic: the eco signal goes back to the transducer and is visualized on a screen as a luminous pattern (Semczyszyn 2010). Yet in $3 \mathrm{D}$ ultrasound, the conversion of the signal into the image might undermine the consistency of the data by adding elements in the picture that are not naturally present in the signal data, such as light and shadows. The question then becomes whether this intermediate step between data acquisition and image rendering (i.e. the mathematical elaboration) interferes with the epistemic value of 3D images.

\section{D ultrasound and computer modelling}

The first step to address the issue is to briefly describe how 3D surface rendered ultrasound images are produced compared with $2 \mathrm{D}$ ultrasound. After the ultrasound signal is received by the transducer, the acquired multiple bi-dimensional sections of the foetus are "frozen" and combined together to form a volumetric record. The data is then mathematically processed and modelled using ray casting algorithms for volume rendering that are capable of reconstructing 3D models from 2D images. The rendering of the shape and surface of the foetus is obtained by exploiting the connection between areas with different echogenic levels (i.e. the foetus with 
high levels and the amniotic fluid with low levels). The ray casting algorithms locate the areas with an abrupt change in echogenicity - where the foetus meets the amniotic fluid - and calculate and model the shape and surface of the foetus. "This is similar to measuring hundreds of points around the foetus and then plotting them into a 3D volume" (Semczyszyn 2010). The image that is generated after this intense mathematical processing is a $3 \mathrm{D}$ model of the foetus (very similar to a highly sophisticated and realistic computer animation), based on biological indicators and the information carried by the echo signal, that captures features of the visual appearance of the foetus (Gee et al. 2004; Scheipers et al. 2010; Solberg et al. 2007; Tonni et al. 2015). Ray casting algorithms are like measuring devices, designed to increase visibility of certain features of the object that are carried by the data (such as the surface) but which are not visible in a normal 2D scan. One of the most interesting features of $3 \mathrm{D}$ foetal imaging is the use of a particular rendering for lighting and shadowing effects. Using this, the facial plane, the limbs, and the entire surface of the foetus are shadowed as if there was a light shining over it. The shadowing is also made using ray casting algorithms that calculate the precise curves and shapes of the foetus and represent them as lighter or darker depending on their distance from the screen and from the angle they are located according to the light source positioned in the image. For this rendering, 3D ultrasound uses computer modelling software (Roth 1982), taken from video game rendering and 3D model reconstruction in architecture, that exploits conventional representational schemes (e.g. linear perspective) to realistically represent the foetus. The main problem with this feature in the image rendering is its inaccuracy. Light is in no way involved in the ultrasound process and sound waves carry no information about light or surface reflectance, which is the reason such graphic rendering of the foetus is intrinsically inaccurate (Gee et al. 2004; Semczyszyn 2010).

As one of the few authors who has analysed its status, Semczyszyn argues that, like drawings, 3D ultrasound is based on appearances. In fact, the visual properties that help us observe things 
in drawings are the one carried by the light and reflected off the surfaces of the object, such as shadowing, surface shape, reflectance, and thickness. Shadows in this context are perceptual markers that allow the viewer to identify and recognize the features of a represented object. 3D ultrasound does exactly this in that it translates the representational scheme signal/luminosity of B-Mode ultrasound into one that reproduces surface reflectance and, at the same time, exploits known perceptual cues and representational schemes like shadowing, surface reflectance, and depth perception (Semczyszyn 2010). The way the volume rendering software is designed, allows 3D ultrasound scans to show the features of the foetus in a way that is more similar to how objects are normally perceived in everyday life, with light, shadows and perspective. For this reason, the observer recognises more easily what is represented in a 3D image rather than in a $2 \mathrm{D}$ ultrasound scan in which the foetus is unfamiliarly represented as dissected $^{3}$. For this reason, 3D ultrasound scans are easier to read than 2D scans yet they seem to deliver information that is in some way arbitrary and contingent. This is because the choice of adding light, shadows and colour to the image is taken arbitrarily by the software developers to make the image more recognizable. The data from the signal does not imply any information about such features, as they are added subsequently in order to simulate ordinary perception.

\section{Modelling interference and epistemic consistency}

We previously defined the epistemic value of images like photographs and MPIs as the capacity of delivering objective information about their depicted subjects. While 3D ultrasound delivers visual information about their subject, information that is even more accessible to the observer than with other typologies of images (such as 2D ultrasound scans), it is unclear to what extent such information is accurate and therefore whether the volume rendering schemes preserve

\footnotetext{
${ }^{3}$ It has been noted in the literature regarding virtual reality (VR) that VR simulation of objects is able grasp the complexity of the perceptual experience of everyday physical objects resulting in the generation of beliefs of veracity in the observer, that transcend other means of representation such as photographs or paintings (Brey 1999). It is not inconsistent to claim that 3D ultrasound models of the foetus are produced precisely to grasp this perceptual complexity.
} 
objectivity. Transforming the echogenic properties to volume rendering involves a significant translation of information: the signal is so altered in the volume rendering procedure that, despite the fact that the image is modelled over it, the standard signal of $2 \mathrm{D}$ ultrasound is no longer available to the viewer (Semczyszyn 2010).

Firstly, the modification of the signal during the volume rendering process makes it difficult to claim that 3D images are MPIs (as we can with 2D ultrasound). On the one hand, 3D ultrasound scans are produced by a fully mechanical and automated process, and are images of a physical specimen. This means that, at least at a level of signal acquisition, there is a direct interaction between the specimen and the machinery. However, the final image is only produced after intense mathematical elaboration, the processing of which interrupts the direct connection between the specimen and the recorded output (the image), which is a criterion of MPI characterization (Perini 2012b).

Secondly, at a more epistemic level, images like 3D ultrasound are capable of conveying objective visual information about the subject they depict. They are crafted and modelled on the echo signal which, as we have described, is capable of delivering information on the shape and size of the foetus. Therefore, these images meet many of the criteria set for the epistemics of MPIs, that is automatic production, causal and counterfactual dependence on the subject they depict, and belief independence. On the other hand, these images also convey information about features - lighting, shadowing effects and colour - all of which are "fabricated" through the rendering process. Another fundamental characterization for MPIs' epistemic status is that the produced image is a visible array of what the machine is "selectively sensitive to" (Perini 2012b, 161). In ultrasound imaging, as we have seen earlier, sound waves are selectively sensitive to the density and shape of tissues, and not to properties carried by light, such as shadows and surface reflectance. Yet the resulting 3D image, through the rendering process, portrays the foetus as hit by a source of light and convey information about features that would be visible 
only if the signal embedded information about light itself. This is only possible thanks to the mathematical processing performed by sophisticated rendering software designed to represent the foetus as if it was seen with ordinary perception. As a result, the observer will form perceptual beliefs regarding the features of the depicted object (the foetus) based on an image that is partly crafted using information embedded in the ultrasound signal (for features like shape and size), as well as using inputs and calculations that are inserted arbitrarily through three-dimensional rendering software (for features like the position of a light source, the shadowing, and the colour of the skin).

One way to approach the issue of discrepancy between objectivity and arbitrariness in 3D ultrasound imaging, is firstly to briefly note that the algorithms used in volume rendering are designed to maintain picture objectivity and aid detection and diagnosis (Semczyszyn 2010). The aim of these images and the reason why these images are produced and continuously enhanced is to make ultrasound images easier to read and interpret without losing their epistemic value in the process. For this reason, the algorithms used to process $3 \mathrm{D}$ images are initially applied to the $2 \mathrm{D}$ signal in a fully automated way and are designed to maintain causal and counterfactual dependency from the original 2D images of the foetus, even when light and shadow are inserted. This is done to preserve information and thus objectivity (Tonni et al. 2015). Algorithms, as we mentioned earlier, are information-preserving calculations designed to objectively and automatically produce an image regardless on the sonographer's beliefs on the represented object. It must be taken into account, however, that these calculations are not always one hundred percent reliable. Sometimes, during the rendering process, the data is mistakenly processed giving rise to a non-reliable image as sources of error may affect volume estimation, such as poor image quality of the original 2D data set, movement artefacts, and incorrect tracing of contours (Blaas et al. 2006). Research is being conducted to advance the reliability of 3D imaging in order to deal with this issue (Hur et al. 2015). 
One might argue that even if the image is fully automated and has a high degree of objectivity guaranteed by standardized mathematical processing, some elements are still inserted quite arbitrarily into the 3D image, such as a source of light that illuminates the foetus, the consequent shading, or colour. Although these features increase the accessibility of visual information about the object, they are not representative of the echogenic data. But what impact does this interference in the image have on the beliefs of the observer and therefore on image objectivity?

Returning to the issue of digital photographic imagery, we have seen that the mechanical photographic process, both analogic and digital, is not without interference. We have also seen that limiting the mental involvement in the production of an image to a secondary level by means of a mechanical process can guarantee a high level of objectivity despite involvement of such mental states. Acknowledging that the mechanical photographic process is not without modification or interference might be useful when discussing 3D ultrasounds. The type of interference that comes from the 3D modelling can be addressed at the secondary level of mental state involvement. While the addition of light and colour can have an impact on the viewer's beliefs, it does not create false objectivity of the image. The perceptual information (shape, size etc.) that the observer extracts from the image is based on the original objective signal, which is why we would consider $3 \mathrm{D}$ images as epistemically relevant sources of information of the foetus inside the womb ${ }^{4}$.

\section{Conclusion}

\footnotetext{
${ }^{4}$ There is still a structural issue in comparing the insertion of light and shadows to 3D ultrasound scans. This type of modification is indeed in line with digital photographic alterations made in post processing - light, shadows and colour are often easily modified by Photoshop and can be considered secondary level mental involvements. But the reason why they are intuitively acceptable as minor changes, that do not undermine the image's objectivity, is because they are made on an already existing image that is created by a technology that is sensitive to light. The problem with 3D ultrasound is that the images are not modifications of already existing images, like in digital photography, but the whole process of the image production is the fabrication of a computer animation that imitates visual perception but is based on a signal that is not sensitive to light like visual perception or photography is. As we have seen for digital photography to identify an acceptable limit for digital alteration is a very difficult task (Benovsky 2014), therefore finding it for digital rendering seems even more challenging at this point.
} 
In this paper, we have focused on defining the epistemic significance of ultrasound scans. We noted the consistency, straightforwardness, and consensus in the literature, in comparing bidimensional ultrasound and photography, both at an ontological and epistemic level. We have shown that B-mode ultrasound produces images mechanically and possess a high epistemic and evidential status that come from their capacity to deliver objective visual information about the objects they represent - in this case, the foetus.

Yet major issues arise when discussing 3D ultrasound scans. This is because they present some epistemic and structural issues that 2D ultrasound do not possess due to the intensive mathematical processing that takes place during the image's production. It is still unclear whether the volume rendering processing preserves the objectivity carried by bi-dimensional ultrasound or whether it creates interference that undermines the epistemic status of 3D images. As image rendering software increases, both the diagnostic capacities and realism of foetal imagine of 3D ultrasound also increase (Tonni, Grisolia, et al. 2016). Despite the epistemic difference we identified between 2D and 3D ultrasound scans it is unclear whether this difference has any clinical significance. Therefore, we propose that further work is required to provide a more concrete characterization and evaluation of the epistemic status of 3D images, and to understand if the different epistemic value of three-dimensional imaging confers any additional diagnostic value to $3 \mathrm{D}$ scans. To this end, a re-examination of the volume rendering interference in ultrasound images is necessary in order to fully characterize these very sophisticated images.

Increasing technological advancements and sophistication in obstetric imaging are challenging the ethical debate with new and prominent interrogations (Edvardsson et al. 2015; Roberts 2012). Subsequently, analysis of the emotional and ethical significance of these images is required. Images are powerful objects, loaded with social and emotional significance, that are capable of shifting our attitudes toward the subjects they represent. It will be interesting to 
analyse the phenomenology of 3D ultrasound scans to investigate how their visualization relates to ethical responsiveness (Mills 2011) and to analyse if and, consequently how, the realistic observation of the foetus through the image can change our attitude towards it. The ethical issues associated with the hyper-realistic visualization of the foetus primarily relate to the conceptualization of the foetus as an autonomous subject, with a singular meaning. Ultrasound scans, as Mills indicates, "put us in relation to a being that we do not otherwise have such a relationship with" (Mills 2011, 103) and the more realistic these images become the more this "being" gains reality as an individual. Some claim that this increased personification of the foetus, due to the sophistication of imaging technologies, together with developments in foetal medicine, has led to the conceptualization of the foetus as a patient in its own right by healthcare professionals. This may lead to ethical dilemmas where the autonomy and identity of the pregnant woman and the unborn child in terms of care, once deeply intertwined, become uncoupled (Zechmeister 2001; Edvardsson et al. 2015). In addition, there are many questions about the impact that visualization of the foetus may have on the decision-making process in cases of pregnancies with foetal abnormalities (Bijma, van der Heide, and Wildschut 2008). These include whether the image of the unborn child actually has an emotional and ethical impact on parents and whether the increasingly clearer visualization of the foetus impacts on the decision to interrupt a pregnancy in the case of severe malformations. As again Mills rightfully noted, ultrasound scans might have profound implications on our perceptions of the ethical status of the human foetus. Three-dimensional images are even capable of showing a realistic portrayal of the face of the child-to-be and the importance of the face within ethics has already been elaborated by Emmanuel Levinas (Derrida 1999). The visualisation of the face of an unborn child might thus create a strong emotional bond between the viewer and the represented foetus, a bond that may challenge society's position on critical matters such as abortion, care, patient's autonomy and personal identity (Gilbert and Howes-Mischel 2004; 
Mills 2011). While all of these issues demand additional thorough consideration as technological advancements in sonography are increasing the realism of ultrasound scans and our ability to clearly see the foetus inside the womb, consideration of these go beyond the scope of this paper.

For the moment, our structural investigation, combined with existing studies on the impact of ultrasonography on pregnant women (Roberts 2012; Black 1992; Simonsen and Rose 2008), provide the first steps towards a comprehensive study on the phenomenology of 3D ultrasound scans.

\section{References}

Alcaraz, Aleksandra Łukaszewicz. 2016. "Epistemic Function and Ontology of Analog and Digital Images." Contemporary Aesthetics 13: 1-14.

Benovsky, Jiri. 2014. “The Limits of Photography.” International Journal of Philosophical Studies 22 (5). Routledge: 716-33. doi:10.1080/09672559.2014.923015.

Bijma, Hilmar H., Agnes van der Heide, and Hajo IJ Wildschut. 2008. “Decision-Making after Ultrasound Diagnosis of Fetal Abnormality." Reproductive Health Matters 16 (31): 82-89. doi:10.1016/S0968-8080(08)31372-X.

Blaas, H. G K, P. Taipale, H. Torp, and S. H. Eik-Nes. 2006. “Three-Dimensional Ultrasound Volume Calculations of Human Embryos and Young Fetuses: A Study on the Volumetry of Compound Structures and Its Reproducibility." Ultrasound in Obstetrics and Gynecology 27 (6): 640-46. doi:10.1002/uog.2794.

Black, Rita Beck. 1992. "Seeing the Baby: The Impact of Ultrasound Technology.” Journal of Genetic Counseling 1 (1): 45-54. 
Boucher, Joanne. 2004. "Ultrasound: A Window to the Womb?: Obstetric Ultrasound and the Abortion Rights Debate.” Journal of Medical Humanities 25 (1): 7-19.

Brey, Philip. 1999. "The Ethics of Representation and Action in Virtual Reality." Ethics and Information Technology 1 (1): 5-14. doi:10.1023/A:1010069907461.

Cohen, Jonathan, and Aaron Meskin. 2004. "On the Epistemic Value of Photographs.” The Journal of Aesthetics and Art Criticism 62 (2): 197-210.

Currie, Gregory. 1991. "Photography, Painting and Perception.” The Journal of Aesthetics and Art Criticism 49 (1): 23-29. doi:10.2307/431646.

Daston, Lorraine, and Peter Galison. 2007. Objectivity. Boston: MIT Press.

Delehanty, Megan Catherine. 2005. "Empiricism and the Epistemic Status of Imaging Technologies.” PhD diss., University of Pittsburgh.

Derrida, Jacques. 1999. Adieu to Emmanuel Levinas. Stanford University Press.

Dretske, Fred. 1981. Knowledge and the Flow of Information. Cambridge MA: MIT Press.

Edvardsson, Kristina, Rhonda Small, Ann Lalos, Margareta Persson, and Ingrid Mogren. 2015. 'Ultrasound's 'Window on the Womb' Brings Ethical Challenges for Balancing Maternal and Fetal Health Interests: Obstetricians' Experiences in Australia.” BMC Medical Ethics 16: 31. doi:10.1186/s12910-015-0023-y.

Gaut, Berys. 2008. “Opaque Pictures.” Revue Internationale de Philosophie 62: 381-95.

Gee, A., R. Prager, G. Treece, C. Cash, and L. Berman. 2004. "Processing and Visualizing Three-Dimensional Ultrasound Data.” British Journal of Radiology 77 (SPEC. ISS. 2). doi:10.1259/bjr/80676194.

Gilbert, S F, and R Howes-Mischel. 2004. "Show Me Your Original Face before You Were 
Born: The Convergence of Public Fetuses and Sacred DNA." History and Philosophy of the Life Sciences 26 (3-4): 377-94.

Hopkins, Robert. 2012. "Factive Pictorial Experience: What's Special about Photographs?" Nous 46 (4): 709-31. doi:10.1111/j.1468-0068.2010.00800.x.

Hur, Hyewon, Young Han Kim, Hee Young Cho, Yong Won Park, Hye-sung Won, Mi-young Lee, Bo Hyon Yun, et al. 2015. "Feasibility of Three-Dimensional Reconstruction and Automated Measurement of Fetal Long Bones Using 5D Long Bone.” Obstetrics and Gynecology Science 58 (4): 268-76.

Lubell, Sam. 2004. "The Womb as Photo Studio.” The New York Times, September 23. http://www.nytimes.com/2004/09/23/technology/circuits/the-womb-as-photostudio.html?mcubz=3.

Lumley, Judith. 1990. “Through a Glass Darkly: Ultrasound and Prenatal Bonding.” Birth 17:4 (December): 214-17.

Martin, Edwin. 1986. “On Seeing Walton's Great-Grandfather.” Critical Inquiry 12: 796-800. Meskin, Aaron, and Jonathan Cohen. 2007. "Photographs as Evidence.” In Photography and Philosophy: Essays on the Pencil of Nature, edited by Scott Walden, 1-17. Oxford, UK: Blackwell Publishing Ltd.

Mills, Catherine. 2011. "Ultrasound, Embodiment and Abortion.” In Futures of Reproduction, 101-21. Springer Netherlands. doi:10.1007/978-94-007-1427-4.

Nanay, Bence. 2010. “Transparency and Sensorimotor Contingencies: Do We See through Photographs?” Pacific Philosophical Quarterly 91 (4): 463-80. doi:10.1111/j.14680114.2010.01380.x.

Perini, Laura. 2012a. "Depiction, Detection, and the Epistemic Value of Photography." The 
Journal of Aesthetics and Art Criticism 70 (1): 913-26.

2012b. "Image Interpretation: Bridging the Gap from Mechanically Produced Image to Representation.” International Studies in the Philosophy of Science 26 (2): 153-70. doi:10.1080/02698595.2012.703478.

Petchesky, Rosalind Pollak. 1987. "Fetal Images : The Power of Visual Culture in the Politics of Reproduction.” Feminist Studies 13 (2): 263-92.

Ramón y Cajal, C L, and R O Martinez. 2005. "Prenatal Observation of Fetal Defecation Using Four-Dimensional Ultrasonography." Ultrasound in Obstetrics and Gynecology 26 (7): 794-95.

Roberts, Julie. 2012. “'Wakey Wakey Baby’: Narrating Four-Dimensional (4D) Bonding Scans.” Sociology of Health and Illness 34 (2): 299-314. doi:10.1111/j.14679566.2011.01345.x.

Roskies, Adina L. 2007. “Are Neuroimages Like Photographs of the Brain?” Philosophy of Science 74 (December): 860-72.

—. 2008. "Neuroimaging and Inferential Distance." Neuroethics 1: 19-30. doi:10.1007/s12152-007-9003-3.

Roth, S D. 1982. "Ray Casting for Modelling Solids.” Computer Graphics and Image Processing 18 (2): 109-44. doi:http://dx.doi.org/10.1016/0146-664X(82)90169-1.

Savendoff, Barbara E. 1997. "Escaping Reality: Digital Imagery and the Resources of Photography." The Journal of Aesthetics and Art Criticism 55 (2): 201-14.

Scheipers, Ulrich, Sergei Koptenko, Rachel Remlinger, Tony Falco, and Martin Lachaine. 2010. “3-D Ultrasound Volume Reconstruction Using the Direct Frame Interpolation Method." IEEE Transactions on Ultrasonics, Ferroelectrics, and Frequency Control 57 
(11): 2460-70. doi:10.1109/TUFFC.2010.1712.

Semczyszyn, Nola. 2010. "Signal into Vision: Medical Imaging as Instrumentally Aided Perception.” PhD diss., University of British Columbia.

Sepulveda, Waldo, Amy E Wong, Francisco Sepulveda, and Renato Ximenes. 2012. "Fetal Magnetic Resonance Imaging and Three-Dimensional Ultrasound in Clinical Practice : General Aspects.” Best Practice \& Research Clinical Obstetrics \& Gynaecology 26 (5). Elsevier Ltd: 575-91. doi:10.1016/j.bpobgyn.2012.05.010.

Simonsen, Sara Ellis, and Nancy C Rose. 2008. "The Complexity of Fetal Imaging Reconciling Clinical Care With Patient Entertainment." Obstetrics and Gynecology 112 (6): 1351-54.

Solberg, Ole Vegard, Frank Lindseth, Hans Torp, Richard E. Blake, and Toril A. Nagelhus Hernes. 2007. "Freehand 3D Ultrasound Reconstruction Algorithms-A Review." Ultrasound in Medicine and Biology 33 (7): 991-1009. doi:10.1016/j.ultrasmedbio.2007.02.015.

Szabo, Thomas L. 2004. Diagnostic Ultrasound Imaging: Inside out. Academic Press.

Taylor, Janelle. 2000. “Of Sonograms and Baby Prams: Prenatal Diagnosis, Pregnancy, and Consumption.” Feminist Studies 26 (2): 391-418.

Tonni, Gabriele, Anna Pia Castigliego, Gianpaolo Grisolia, Mario Lituania, Simon Meagher, Fabrício Da, Silva Costa, and Edward Araujo Júnior. 2016. “Three-Dimensional Ultrasonography by Means of HDlive Rendering in the First Trimester of Pregnancy : A Pictorial Review." Journal of the TurkishGerman Gynecological Association 17: 11019. doi:10.5152/jtgga.2016.15201.

Tonni, Gabriele, Gianpaolo Grisolia, Eduardo Félix Santana, and Edward Araujo Junior. 
2016. “Assessment of Fetus during Second Trimester Ultrasonography Using HDlive Software: What Its Real Application in the Obstetrics Clinical Practice?" World Journal of Radiology 8 (12): 922-28. doi:10.4329/wjr.v8.i12.922.

Tonni, Gabriele, Wellington P. Martins, Hélio Guimarães Filho, and Edward Araujo Júnior. 2015. "Role of 3-D Ultrasound in Clinical Obstetric Practice: Evolution Over 20 Years." Ultrasound in Medicine and Biology 41 (5): 1180-1211. doi:10.1016/j.ultrasmedbio.2014.12.009.

Walden, Scott. 2005. “Objectivity in Photography.” British Journal of Aesthetics 45: 258-72.

Walton, Kendall L. 1984. "Transparent Pictures : On the Nature of Photographic Realism." Critical Inquiry 11 (2): 246-77.

Warburton, Nigel. 1988. "Seeing through 'Seeing through Photographs.”' Ratio 1: 64-74.

Zechmeister, Ingrid. 2001. "Foetal Images: The Power of Visual Technology in Antenatal Care and the Implications for Women's Reproductive Freedom.” Health Care Analysis 9 (4): 387-400. doi:10.1023/A:1013837511115. 